\title{
Correlations Between Depression, Cognitive Status, Functional Scores, Disability and Lesion Load in Multiple Sclerosis Treated with Interferon Beta 1a
}

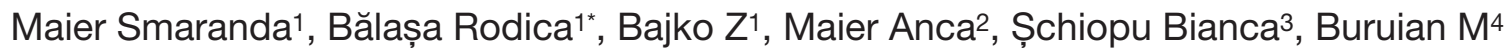 \\ 1 University of Medicine and Pharmacy Târgu Mureș, Department of Neurology \\ 2 Institute for Cardiovascular Diseases and Transplantation TarguMureș, Department of Cardiology \\ ${ }^{3}$ Mureș County Clinical Emergency Hospital TârguMureș, Department of Neurology \\ 4 University of Medicine and Pharmacy TârguMureș, Department of Radiology
}

\begin{abstract}
Introduction: Depression and cognitive impairment are the most frequent mental disorders in multiple sclerosis (MS) and represent an important cause of morbidity and mortality. The aim of the study was to analyse the main determinants of depression in multiple sclerosis.

Materials and methods: Thirty-two patients with relapsing remitting multiple sclerosis (RRMS), treated with Interferon Beta 1a, without relapses and corticosteroid treatment in the last 30 days, were included in the study. The mean age of the patients was $35.4 \pm 9.2 \mathrm{years}, \mathrm{M} / \mathrm{F}$ ratio 0.33. Depression level was evaluated by the Romanian version of Beck Depression Inventory (BDI) and the cognitive function with Paced Auditory Serial Addition Test 3 (PASAT 3), Symbol Digit Modalities Test (SDMT). The functional status and disability level of the patients were evaluated with Multiple Sclerosis Functional Composite and Expanded Disability Status Scale. In all patients a cerebral MRI with intravenous contrast administration was performed using a 1.5T MRI device.

Results: Twenty-three patients were free of depression (score 1-10), 4 patients presented mild mood disturbance (score 11-16), 3 borderline clinical depression (score 17-20) and 2 moderate depression (score 21-30). The mean BDI score was 8.71 \pm 7.16 . BDI score correlated significantly with EDSS ( $R=0.38, p=0.03)$, PASAT $3(R=-0.42, p=0.01)$, SDMT ( $R=-0.58, p=0.0007)$, Timed 25-Foot Walk ( $R=0.43$, $p=0.01$ ) and 9-Hole Peg Test $(R=0.45, p=0.008)$. From the EDSS functional scores, significant correlations were found with the urinary score $(R=0.4$, $p=0.01)$ and sensitive score $(R=0.49, p=0.004)$. BDI score correlated significantly with the total number of $T 2$ lesions $(R=0.31, p=0.05)$ while there was no correlation with the number of active lesions.

Conclusions: The main determinants of depression in RRMS patients are the cognitive impairment, the affection of fine hand movements (9-HP), gait impairment (T25FT) and bladder and sensitive dysfunction.
\end{abstract}

Keywords: multiple sclerosis, depression, disability, EDSS, functional scores

Received: 08 December 2014 / Accepted: 12 January 2015

\section{Introduction}

Multiple sclerosis (MS) is a chronic, immune-mediated, multifocal inflammatory disease of the central nervous system that attacks myelinated axons, producing variable destruction of myelin and axons and hence significant physical disability. (1)

MS is the most common cause of severe neurological disability in young adults in the Western world (being usually diagnosed between 20 and 40 years of age), presenting with various and unpredictable symptoms that may include weakness, visual impairment, sensory problems, bowel and bladder incontinence, imbalance or pain in various parts of the body. The time course of the disease is uncertain. (2)

In the recent decades there has been increasing interest in cognitive impairment and mood disorders in MS, as well as the physical disabilities associated with the disease. (3)

Charcot was the first person to describe psychiatric symptoms in patients with MS in lectures at the Salpetriere hospital in the 19th century; Charcot described

Correspondence to: Rodica Bălașa

E-mail: iipascu@redatronic.ro mania, hallucinations and depression alongside other signs of the disease. Depression is the most frequent psychiatric diagnosis in patients with MS and is an important source of morbidity and mortality. Lifetime prevalence of major depression ranges from 19 to $54 \%$, three times higher than in the general population. Published data put the annual prevalence of depression at $16 \%$ and the prevalence of clinically significant depressive symptoms at 50\%. Risk factors for major depression in MS include female gender, age less than 35 years, family history of major depression and stress. $(4,5)$

An investigation of the relationship between the frequency and severity of depression and the degree of functional disability suggested that patients with an elevated Expanded Disability Status Scale (EDSS) score were more likely to develop clinically significant depression. (6)

Recent studies found that depression is frequently disregarded and undertreated in patients with MS; the diagnosis was missed in about $23-30 \%$ of the cases and inadequately treated in $20-36 \%$ of reported cases. (4)

The degree of cognitive impairment is one of the most important determinants of quality of life of patients with MS. Neuropsychological studies suggest that $40-65 \%$ of 
MS patients develop a cognitive impairment, impairments range from mild short-term memory deficits to severe impairments such as dementia. (7)

Cognitive impairment may occur at any stage of the disease, including clinically isolated syndrome and early relapsing-remitting multiple sclerosis (RRMS). The areas of cognition most affected in MS patients are information processing speed, episodic memory and executive function. (8)

The aim of this study was to evaluate depression and cognitive function in MS patients and calculate the correlations between measures of depression, cognitive status, functioning, disability and lesion load in MS.

\section{Materials and methods}

Thirty-two RRMS patients treated with Interferon Beta 1a were included in the study. The inclusion criteria were: 1 . male or female 18 - 55 years old; 2. diagnosis of RRMS according to modified McDonald criteria 2010 with diseasemodifying therapies (DMT) indicated; 3. Patient is immunomodulator-naïve to drugs other than Interferon beta; 4. (female subjects only) practising effective contraception unless postmenopausal or surgically sterile; 5 . able to comply with trial protocol; 6 . able to undergo MRI. The exclusion criteria were: 1 . treatment with corticosteroids within 30 days of testing. 2. participation in another non-MS trial within 3 months of the start date. 3. presence of a severe concomitant condition that is likely to affect participation in the study; 4. neuropsychiatric disorders unrelated to the MS; 5. physical impairment that could interfere with cognitive testing.

Depression level was evaluated using the Romanian version of Beck Depression Inventory (BDI); cognitive function with the Paced Auditory Serial Addition Test 3 (PASAT 3). Patients' functional status and disability level were evaluated with the Multiple Sclerosis Functional Composite (MSFC) and Expanded Disability Status Scale (EDSS). All patients underwent cerebral MRI with a 1.5T MRI device, with intravenous administration of a contrast agent.

The BDI consists of 21 questions; responses are given on a four-point severity scale. A BDI score between 1 and 10 is considered normal, a score between 11 and 16 indicates mild mood disorder, 17 - 20 indicates borderline depression, 21 - 30 indicates mild depression, 31 - 40 indicates severe depression and a score above 40 indicates extremely severe depression. (9)

EDSS is a unified scale for the evaluation of functioning in MS patients; it assesses impairment in 8 functional systems. Total score on the EDSS can be between 0.0 (normal neurological status) to up to 10.0 (death). (10)

The Symbol Digit Modalities Test (SDMT) was used to assess information processing speed. The test consists of single digits paired with abstract symbols. Rows of 9 symbols are arranged randomly, and the patient must say or write the number paired with the symbol displayed. The score was given by the total number of correct responses in
90 seconds (range $0-110$ ). The SDMT is sensitive to cognitive decline and in clinical studies it has been shown to be one of the best predictors of secondary cognitive impairment in MS. (11-12)

The MSFC was used to evaluate three important clinical features of MS: leg function/ambulation (Timed 25-Foot Walk; T25-FW), hand function (9-Hole Peg Test; 9-HPT) and cognitive status (PASAT). Scores on each component are converted to standard scores. The T25-FW was the first MSFC component administered at each visit and provided a quantitative measure of leg function. The test requires the patient to walk 25 feet as quickly and safely as possible, following a clearly marked 25 -foot course; the examiner measures and records the time taken. The test is performed twice. The 9-HPT is the second MSFC component administered. The 9-HPT is a quantitative measure of hand function; both the dominant and non-dominant hand were tested twice. (13-14)

The PASAT, the third MSFC component, was a measure of cognitive status based on tests of auditory information processing speed and calculation ability. The PASAT is presented on compact disc so that the rate of stimulus presentation can be controlled by the experimenter. One digit is presented every 3 minutes; the patient must add the new digit to the immediately preceding digit, and the score is the number of correct answers (maximum $=60)$. (13-14).

MRI examinations were performed with a 1.5 Tesla MRI device (GE Signa Excite II). The MRI protocol included the following sequences: T1-3D-FSPGR axial native+gadolinium, T2-SE axial, FLAIR axial, FLAIR sagittal, double inversion recovery (DIR) axial, magnetisation transfer imaging (MTI) axial and diffusion tensor imaging (DTI) axial.

\section{Statistical analysis}

Differences between groups in terms of outcome variables were described using standard statistics, evaluated for significance using two-sample t-tests or Wilcoxon's rank-sum tests, depending on whether the distributional assumptions were satisfied judging by skewness-kurtosis tests.

Correlations between continuous variables were evaluated by calculating Pearson's or Spearman's coefficients depending on whether the linearity assumption was satisfied. Coefficients were estimated complete with $95 \%$ confidence intervals (based on Fisher's transformation) and $p$ values, both for the whole sample and stratified for patient group.

\section{Results}

Demographic data for the patients are presented in Table I. Twenty-three patients showed no symptoms of depression (BDI score 1-10), 4 patients had mild mood disturbance (BDI score 11-16), 3 had borderline clinical depression (BDI score 17-20), and 2 had moderate depression (BDI score 21-30). The mean BDI score was 8.71 \pm 7.16 . There was no statistically significant sex difference in BDI scores. 
Table I. Demographic characteristics, clinical and imagistic data of the patient group.

\begin{tabular}{lc}
\hline & All Patients $\mathbf{n}=\mathbf{3 2}$ \\
\hline Age at onset of MS (years) & $29.07 \pm 8.72$ \\
\hline Age at testing (years) & $35.44 \pm 9.19$ \\
\hline Treatment duration (years) & $3.35 \pm 3.12$ \\
\hline MS duration (years) & $6.37 \pm 3.71$ \\
\hline Gender Men/Women & $7 / 25$ \\
\hline EDSS at start of treatment & $1.42 \pm 0.6$ \\
\hline EDSS at testing & $1.67 \pm 1.64$ \\
\hline Number of relapses in the previous year & $0.63 \pm 0.79$ \\
\hline Number of T2 lesions & $24.28 \pm 17.1$ \\
\hline Number of active lesions in T1 & $0.21 \pm 0.35$
\end{tabular}

BDI score correlated significantly with scores on the EDSS $(\mathrm{R}=0.38, \mathrm{p}=0.03)$; Figure 1), PASAT $3(\mathrm{R}=-0.42$, $\mathrm{p}=0.01$; Figure 2$)$, SDMT $(\mathrm{R}=-0.58, \mathrm{p}=0.0007$; Figure 3$)$, $\mathrm{T} 25-\mathrm{FW}(\mathrm{R}=0.43, \mathrm{p}=0.01$; Figure 4$)$ and 9-HPT $(\mathrm{R}=0.45$, $\mathrm{p}=0.008$; Figure 5).

EDSS functional scores correlated with the urinary score $(\mathrm{R}=0.4, \mathrm{p}=0.01)$ and sensitive score $(\mathrm{R}=0.49, \mathrm{p}=0.004)$.
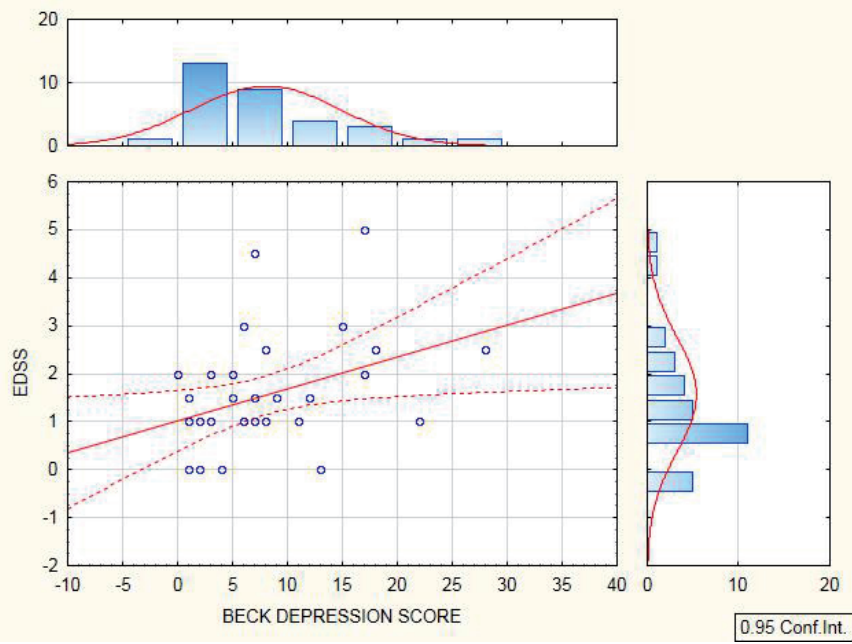

Fig. 1. Correlation between Beck Depression Inventory Scale and EDSS (Spearman $\mathrm{R}=0.38, \mathrm{p}=0.03$ ).
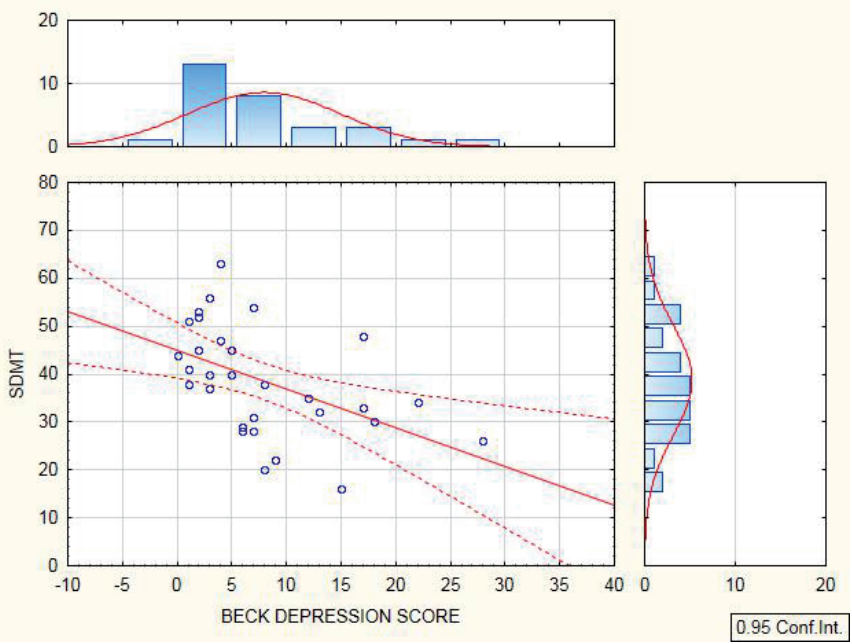

Fig. 3.Correlation between Beck Depression Inventory and SDMT (Spearman R=-0.58, $\mathrm{p}=0.0007$ ).
The other components of EDSS functional scores were not correlated with BDI score.

BDI score correlated significantly with the total number of $T 2$ lesions ( $R=0.31, p=0.05)$ (Figure 6 ) but not with the number of active lesions.

BDI score was not correlated with patient age, duration of disease or number of relapses in the last year.

\section{Discussion}

Evaluations of disability in MS place more emphasis on motor and ambulatory functions than on higher cortical functions; however, there is a wide range of neuropsychiatric comorbidities that significantly affect the quality of life of patients with MS $(6,15,16)$.

The relationship between depression and MS has long been recognised and investigated; most studies have reported high rates of depression in the MS population $(15,17,18)$.

A study conducted in Vancouver, which involved administration of a psychiatric interview to 221 patients with MS, revealed that $34.4 \%$ of patients had a lifetime diagnosis of depression and $50.3 \%$ of patients were estimated
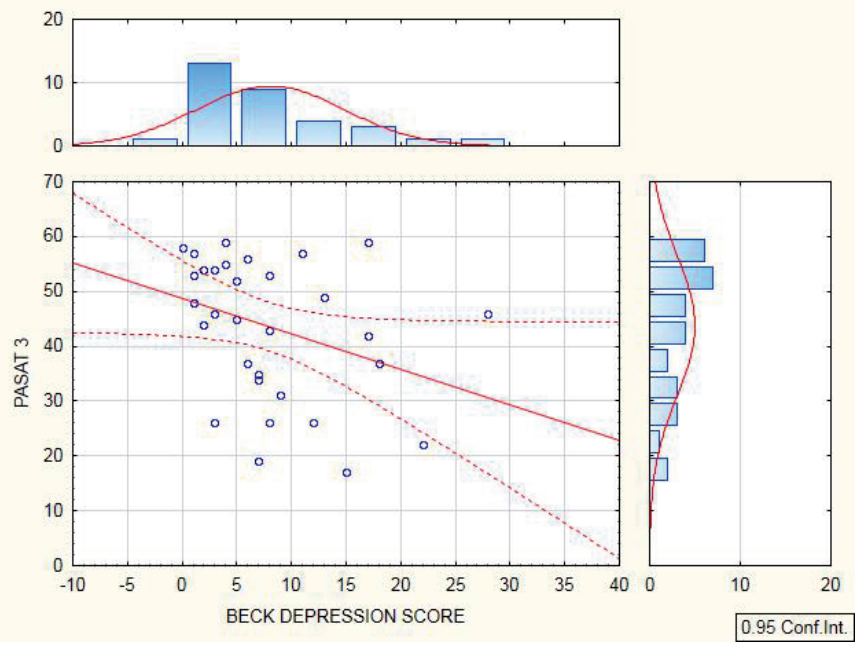

Fig. 2.Correlation between Beck Depression Inventory and PASAT 3 (Spearman $\mathrm{R}=-0.42, \mathrm{p}=0.01$ ).

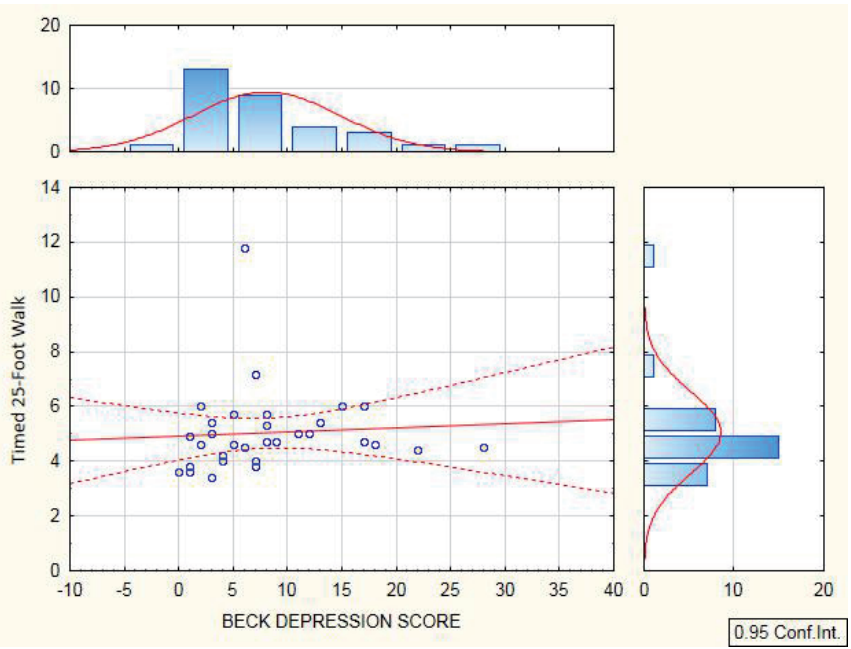

Fig. 4.Correlation between Beck Depression Inventory and T25FW (Spearman $\mathrm{R}=0.43, \mathrm{p}=0.01$ ). 


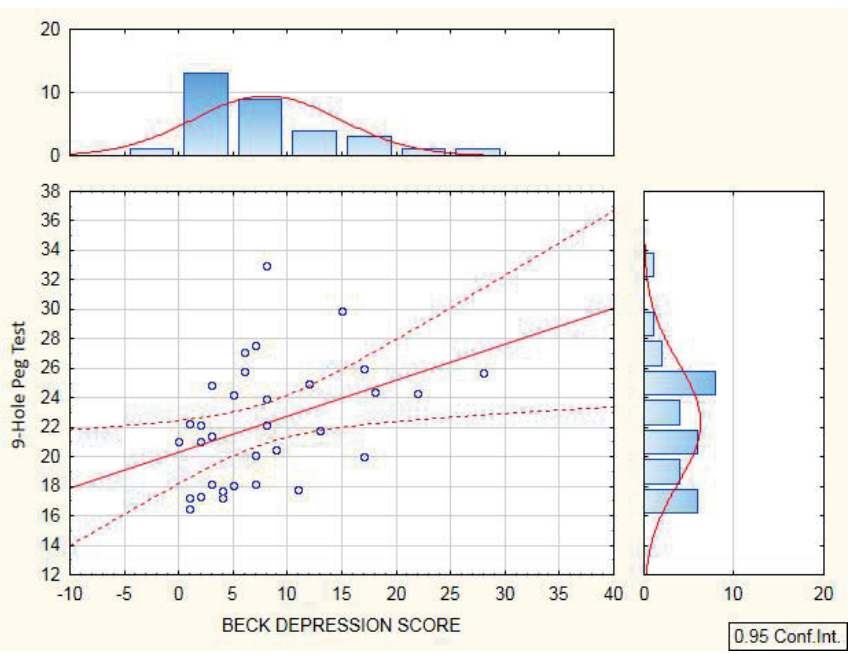

Fig. 5.Correlation between Beck Depression Inventory and 9HPT (Spearman $\mathrm{R}=0.45, \mathrm{p}=0.008$ ).

to be at risk for developing depression by age of 59 years $[15,19]$. A Canadian study reported a $42 \%$ lifetime prevalence of clinically significant depression in patients with MS $[15,20]$.

Minden et al. concluded that $54 \%$ of MS patients [50] in their sample had met the diagnostic criteria for major depression at least once since their diagnosis; $14 \%$ of this subgroup had met the criteria prior to diagnosis $[15,21]$.

Schiffer found that up to $65 \%$ of patients with MS reported depressive symptoms [15,22].

There have been few epidemiological studies of depression in MS. One population-based study examined 115,071 patients aged 18 years and older using the Composite International Diagnostic Interview Short Form for Major Depression. The one-year prevalence of major depression was $25.7 \%$ in the 322 participants with MS compared with only $8.9 \%$ in the general population $[15,21]$.

Depression in MS may be more than a simple reaction to this debilitating disease; structural, psychoneuroimmunological and neurochemical abnormalities also play an important role. Understanding why depression is so frequent in MS patients is a complex and challenging task. The high incidence of depression in MS cannot simply be attributed to the burden of living with a disabling neurological disorder because there are other more severe neurological diseases, such as amyotrophic lateral sclerosis, in which depression is less frequent $[15,23]$.

One might expect a linear relationship between depression and functional or physical disability in MS; however this has not been observed in all relevant studies. The reported lack of correlation between functional disability and depression suggests that the depression has other biological causes. The location of lesions in patients with MS and the degree to which limbic circuits are affected may represent a more important aetiological factor in depression in MS than physical impairment. One study found a significant correlation between depression and major cognitive decline and quality of life scores; surprisingly, depression

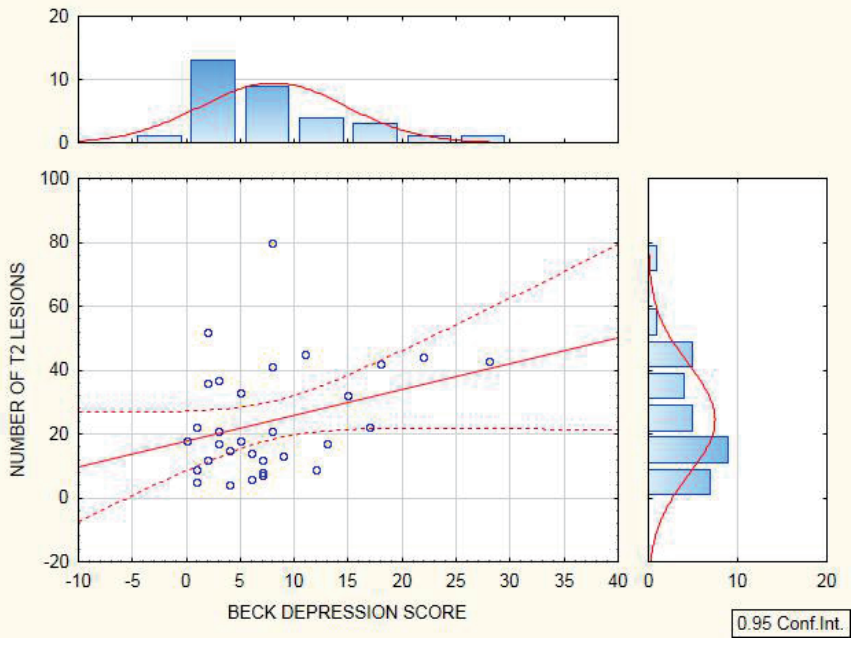

Fig. 6.Correlation Between Beck Depression Inventory and total number of $\mathrm{T} 2$ lesions (Spearman $\mathrm{R}=0.31, \mathrm{p}=0.05$ ).

was not correlated with neurological disability [15,24,25]. However, other authors found at least some correlation between physical functioning and depression in MS [15,26].

A study of $50 \mathrm{MS}$ patients attending an outpatient clinic reported that BDI scores increased with disease severity and physical disability but not with disease duration (15, 27-31)

In this study we found a significant correlation between depression and an objective measure of disability, the EDSS. Separate calculations of the correlation between depression and the 8 functional systems of the EDSS revealed that only bowel and bladder dysfunction and sensitive score were correlated with BDI score. Chwastiak et al. reported that the EDSS cognitive function score correlated more strongly with depression than other EDSS scores [6]. We did not replicate this finding. The EDSS cognitive function is based largely on self-report items; PASAT and SDMT scores provide more sensitive and objectives measures of cognitive functioning. We found a significant correlation between BDI score and functional impairment of the lower and upper extremities, assessed by T25-FW and 9-HPT respectively. This is the first report of a correlation between symptoms of depression and objective measures of physical disability.

Some studies suggest that depression is more common in women $[15,32,33]$, but the evidence on the relationships between age or gender and incidence of depression in patients with MS is inconsistent. Several studies found no significant gender-related difference in the incidence of depression $[6,15,34]$. Several community and tertiary centre studies have reported that older patients with MS report fewer symptoms of depression than younger patients $[15,30,35]$. We did not find any correlations between depression and patient age or gender.

The cognitive impairment is another factor that significantly influence life satisfaction and quality of life in MS; it is also the most important determinant of employment status and the social costs associated with MS $[8,36]$. The 
prevalence of cognitive impairment in patients with MS is between $43-70 \%$, cognitive impairment can occur at any stage of the disease and is only weakly correlated with disease duration and disability; some studies suggest that cognitive impairment is strongly associated with certain MRI parameters [8,37-39].

The current evidence for an association between cognitive impairment and depression is inconsistent, the association may be bidirectional [40].

Several studies have found that patients with MS-related cognitive dysfunction report higher levels of depression than patients without cognitive impairment $[24,40,41]$. Other studies have failed to find an association between depression and cognitive impairment [42].

Some studies have suggested that specific types of cognitive impairment may be preferentially related to depression, for example autobiographical memory deficits [43]. We found a significant correlation between BDI scores and scores on two tests known to be sensitive to cognitive impairment, PASAT, a combined measure of auditory information processing speed and calculation ability, and SDMT, a measure of information processing speed

There is little empirical evidence on relationship between lesion burden (assessed by MRI) and depression in MS. Feinstein et al. concluded that the burden of demyelinating lesions was associated with the severity of depression [44]; we found that the level of depression correlated significantly with the number of T2 lesions on an MRI scan.

The main limitation of our study was the small size of the patient sample.

\section{Conclusions}

Our results suggest that there are significant correlations between depression and cognitive impairment, some important aspects of physical disability being assessed using an objective instrument and MRI parameters such as T2 lesion burden. The main determinants of depression in RRMS patients were cognitive impairment, the impairment in fine hand movements (9-HPT score), gait impairment (T25-FT) and bladder and sensitive dysfunction. The evidence for an association between these variables and depression remains confused and further research in this area is needed.

\section{References}

1. Luzzio C. Multiple Sclerosis.eMedicine.Medscape. Retrieved 11/25/2014 from http://emedicine.medscape.com/article/1146199-overview

2. Reese JP, Wienemann G, John A, Linnemann A, Balzer-Geldsetzer M, Mueller UO, Eienbröker C, Tackenberg B, Dodel R. Preference-based Health status in a German outpatient cohort with multiple sclerosis. Health Qual Life Outcomes; 2013; 11:162.

3. Sahraian MA, Etesam F. Cognitive Impairment in Multiple Sclerosis. ZJRMS 2014;16(1):1-7

4. Skokou M, Soubasi E, GourzisP.Depression in multiple sclerosis: a review of assessment and treatment approaches in adult and pediatricpopulations. ISRN Neurol. 2012;2012:427102. doi: 10.5402/2012/427102.

5. Siegert RJ, Abernethy DA. Depression in multiple sclerosis: a review. J NeurolNeurosurg Psychiatry. 2005 Apr;76(4):469-75.
6. Chwastiak L, Ehde DM, Gibbons LE, Sullivan M, Bowen JD, Kraft GH.Depressive symptoms and severity of illness in multiple sclerosis: epidemiologic study of a large community sample. Am J Psychiatry. 2002 Nov;159(11):1862-8.

7. Niino M, Mifune N, Kohriyama T, Mori M, Ohashi T, Kawachi I, Shimizu Y, Fukaura H, Nakashima I, Kusunoki S, Miyamoto K, Yoshida K, Kanda T, Nomura K, Yamamura T, Yoshii F, Kira J, Nakane S, Yokoyama K, Matsui M, Miyazaki Y, Kikuchi S.Apathy/depression, but not subjective fatigue, is related with cognitive dysfunction in patients with multiple sclerosis. BMC Neurol. 2014;14:3. doi: 10.1186/1471-2377-14-3.

8. Langdon DW, Amato MP, Boringa J, Brochet B, Foley F, Fredrikson S, Hämäläinen P, Hartung HP, Krupp L, Penner IK, Reder AT, Benedict $\mathrm{RH}$. Recommendations for a Brief International Cognitive Assessment for Multiple Sclerosis (BICAMS).MultScler. 2012;18(6):891-8. doi: 10.1177/1352458511431076.

9. Beck AT, Ward CH, Mendelson M, Mock J, Erbaugh J. An inventory for measuring depression.Arch Gen Psychiatry.1961;4:561-71.

10. Kurtzke JF. Rating neurologic impairment in multiple sclerosis: an expanded disability status scale (EDSS). Neurology. 1983;33(11):1444-52

11. Parmenter BA, Weinstock-Guttman B, Garg N, Munschauer F, Benedict $\mathrm{RH}$. Screening for cognitive impairment in multiple sclerosis using the Symbol digit Modalities Test. MultScler. 2007 Jan;13(1):52-7.

12. Benedict RH, Duquin JA, Jurgensen S, Rudick RA, Feitcher J, Munschauer FE, Panzara MA, Weinstock-Guttman B. Repeated assessment of neuropsychological deficits in multiple sclerosis using the Symbol Digit Modalities Test and the MS Neuropsychological Screening Questionnaire. Mult Scler. 2008;14(7):940-6. doi: 10.1177/1352458508090923.

13. Cutter GR, Baier ML, Rudick RA, Cookfair DL, Fischer JS, Petkau J, Syndulko K, Weinshenker BG, Antel JP, Confavreux C, Ellison GW, Lublin F, Miller AE, Rao SM, Reingold S, Thompson A, Willoughby E. Development of a multiple sclerosis functional composite as a clinical trial outcome measure. Brain 1999; 122:871-882.

14. Fischer JS, Rudick RA, Cutter GR, Reingold SC. The Multiple Sclerosis Functional Composite Measure (MSFC): an integrated approach to MS clinical outcome assessment. National MS Society Clinical Outcomes Assessment Task Force. MultScler 1999;5(4):244-250.

15. Kanner A. Depression in Neurologic Disorders: Diagnosis and Management. August 2012, Wiley-Blackwell

16. Huijbregts SC, Kalkers NF, de Sonneville LM, et al. Cognitive impair ment and decline in different MS subtypes. J Neurol Sci 2006;245:187-194.

17. Whitlock FA, Siskind MM. Depression as a major symptom of multiple sclerosis. J Neurol Neurosurg Psychiatry 1980;43:861-865.

18. Schubert DS, Foliart RH. Increased de pression in multiple sclerosis patients. A meta - analysis. Psychosomatics 1993;34:124-130.

19. Sadovnick AD, Remick RA, Allen J, et al. Depression and multiple sclerosis. Neurology 1996;46:628-632.

20. Joffe RT, Lippert GP, Gray TA, et al. Mood disorder and multiple sclerosis . Arch Neurol 1987;44:376-378.

21. Patten SB, Beck CA, Williams JV, et al. Major depression in multiple sclerosis: a population - based perspective. Neurology 2003;61:15241527.

22. Schiffer RB. Depression in neurological practice: diagnosis, treatment, implications. Semin Neurol 2009;29:220-233.

23. Rabkin JG, Albert SM, Del Bene ML, et al. Prevalence of depressive disorders and change over time in late - stage ALS. Neurology 2005;65:62-67.

24. Gilchrist AC, Creed FH. Depression, cognitive impair ment and social stress in multiple sclerosis. J Psychosom Res 1994. 38:193-201.

25. Lobentanz IS , Asenbaum S, Vass K, et al. Factors influencing quality of life in multiple sclerosis patients: disability, depressive mood, fatigue and sleep quality. Acta Neurol Scand 2004;110:6 - 13.

26. Janssens AC, van Doorn PA, de Boer JB, et al. Anxiety and depression influence the relation between disability status and quality of life in multiple sclerosis. Mult Scler 2003;9:397-403.

27. Galeazzi GM, Ferrari S, Giaroli G, et al. Psychiatric disorders and depression in multiple sclerosis outpatients: impact of disability and interferon beta therapy. Neurol Sci 2005;26:255-262.

28. Gay MC, Vrignaud P, Garitte C, et al. Predictors of depression in multiple sclerosis patients. Acta Neurol Scand 2010;121:161-170.

29. Dahl OP, Stordal E, Lydersen S, et al. Anxiety and depression in multiple sclerosis. A comparative population - based study in Nord - Trondelag County, Norway. Mult Scler 2009;15:1495-1501.

30. Beal CC, Stuifbergen AK, Brown A. Depression in multiple sclerosis: a longitudinal analysis. Arch Psychiatr Nurs 2007;21:181-191.

31. Zabad RK, Patten SB, Metz LM. The association of depression with disease course in multiple sclerosis. Neurology. 2005;64:359-360. 
32. Kessler RC, McGonagle KA, Swartz M, et al. Sex and depression in the National Comorbidity Survey: lifetime prevalence, chronicity and recurrence. J Affect Disord 1993;29:85-96.

33. Weissman MM, Bland RC, Canino GJ, et al. Cross-national epidemiology of major depression and bipolar disorder. JAMA 1996;276:293-299.

34. Beiske AG, Svensson E , Sandanger I, et al. Depression and anxiety amongst multiple sclerosis patients. Eur J Neurol 2008;15:239-245.

35. Kneebone II, Dunmore EC, Evans E. Symptoms of depression in older adults with multiple sclerosis (MS): comparison with a matched sample of younger adults. Aging Ment Health 2003;7:182-185.

36. Benedict $\mathrm{RH}$, Amato MP, Boringa J, et al. Brief International Cognitive Assessment for MS (BICAMS): international standards for validation. BMC Neurol 2012;16:12:55

37. Amato MP, Portaccio E, Goretti B, et al. Relevance of cognitive deterioration in early relapsing-remitting MS: a 3-year follow-up study. Mult Scler 2010;16:1474-1482.

38. Amato MP, Portaccio E, Stromillo ML, et al. Cognitive assessment and quantitative magnetic resonance metrics can help to identify benign multiple sclerosis. Neurology 2008;71:632-638.
39. Filippi M, Rocca MA, Benedict $\mathrm{RH}$, et al. The contribution of MR in assessing cognitive impairment in multiple sclerosis. Neurology 2010;75:2121-2128.

40. Wallin MT, Wilken JA, Turner AP, Williams RM, Kane R. Depression and multiple sclerosis: Review of a lethal combination. J Rehabil Res Dev 2006;43:45-62.

41. Maor Y, Olmer L, Mozes B. The relation between objective and subjective impairment in cognitive function among multiple sclerosis patients--the role of depression. Mult Scler 2001;7:131-135.

42. Krupp LB, Sliwinski M, Masur DM, Friedberg F, Coyle PK. Cognitive functioning and depression in patients with chronic fatigue syndrome and multiple sclerosis. Arch Neurol 1994;51:705-710.

43. Kenealy PM, Beaumont JG, Lintern TC, Murrell RC. Autobiographical memory in advanced multiple sclerosis: assessment of episodic and personal semantic memory across three time spans. J Int Neuropsychol Soc 2002;8:855-860.

44. Feinstein A, Roy P, Lobaugh N, Feinstein K, O'Connor P, Black S. Structural brain abnormalities in multiple sclerosis patients with major depression. Neurology 2004;62:586-590 